\title{
Supplementary Material: Role of Internal DNA Motion on the Mobility of a Nucleoid Associated Protein
}

\author{
Indresh Yadav, ${ }^{1}$ Rajib Basak, ${ }^{1}$ Peiyan Yan, ${ }^{1}$ Jeroen A. van \\ Kan, ${ }^{1}$ Véronique Arluison, ${ }^{2,3}$ and Johan R.C. van der Maarel ${ }^{1, *}$ \\ ${ }^{1}$ Department of Physics, National University of Singapore, Singapore 117542 \\ ${ }^{2}$ Université de Paris, UFR SDV, 75006 Paris, France \\ ${ }^{3}$ Laboratoire Léon Brillouin, CEA, CNRS, \\ Université Paris Saclay, 91191 Gif-sur-Yvette, France
}

\section{MATERIALS AND METHODS}

\section{Chip fabrication}

Nanofluidic devices featuring rectangular-shaped channels with a length of $90 \mu \mathrm{m}$, a depth of $130 \pm 5 \mathrm{~nm}$, and a width of $120 \pm 5 \mathrm{~nm}$ (125-nm channel system) were fabricated by replication in polydimethylsiloxane with enhanced elasticity modulus (X-PDMS) of a patterned master stamp [1-3]. The nanochannel part of the stamp was made in hydrogen silsesquioxane resist (Dow Corning, Midland, MI) using a lithography process with proton beam writing. An array of nanochannels is connected to two loading reservoirs through a superposing set of microchannels made in mr-DWL photoresist (Micro Resist Technology, Berlin, Germany) with a laser writer (Heidelberg micro PG 101). The heights and widths of the ridges in the master stamp were measured with atomic force microscopy (Dimension 3000, Veeco, Woodbury, NY) and scanning electron microscopy (JEOL JSM6700F), respectively. The master stamp was coated with diamond-like-carbon layer before being copied in the inorganic-organic hybrid polymer OrmoStamp (Micro Resist Technology). After copying, the stamp was coated with a 5-nm-thick carbon layer to ensure perfect release of the replicated chips. The stamp was replicated in X-PDMS followed by curing at $333 \mathrm{~K}$ for $24 \mathrm{~h}$. Following plasma oxidation (Harrick, Ossining, NY), the X-PDMS replica was sealed with a glass coverslip.

\footnotetext{
* johanmaarel@gmail.com
} 


\section{Sample preparation}

Bacteriophage $\lambda$-DNA (48.5 kbp, contour length of $16.5 \mu \mathrm{m})$ was purchased from New England Biolabs, Ipswich, MA. The stock solution has a concentration of $0.5 \mathrm{~g}$ of DNA/L in TE buffer (10 mM Tris-HCl, pH 8.0, and 1 mM EDTA). Covalently bonded concatemers

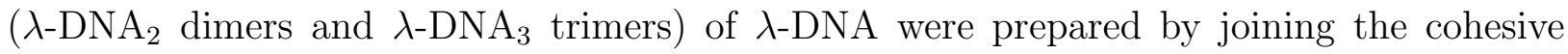
ends through phosphodiester bonds and enzymatic ligation with T4 DNA ligase (Promega, Madison, WI) [4]. The appropriate amounts of DNA and enzyme were mixed and ligation was carried out overnight at $277 \mathrm{~K}$. The reaction was inactivated by heating the sample to $338 \mathrm{~K}$ for $10 \mathrm{~min}$. Finally, the sample was dialysed in a micro-dialyser to remove excess salts and enzyme before re-dispersing in TE buffer. Full length Hfq $\left(M_{w}=67 \mathrm{kDa}\right.$, hexamer $)$ was purified from over-expressing BL21(DE3)/pTE608 cells as previously described [5]. To obtain the fluorescent form of the protein, S38C Hfq was labeled with a Cy3 maleimide reactive-dye (GE-Healthcare, Chicago, IL) [6]. The labeling efficiency is $80 \%$, that is the average number of fluorphores per Hfq hexamer is about five. Solutions of DNA and Hfq were mixed and incubated overnight at $277 \mathrm{~K}$. The final concentrations are $0.3 \mathrm{mg}$ of DNA/L and $0.01 \mathrm{mg}$ of $\mathrm{Hfq} / \mathrm{L}$. Prior to fluorescence imaging, DNA was stained with inter-calating dye YOYO-1 (Invitrogen, Carlsbad, CA, USA) at a ratio of one dye molecule for ten base pairs. No anti-photo bleaching agent was used.

\section{Fluorescence imaging}

The stained DNA/Hfq solution was pipetted into the two loading reservoirs connected by the array of nanochannels. The DNA molecules were subsequently driven into the channels by electrophoresis. For this purpose, two platinum electrodes were immersed in the reservoirs and connected to a power supply with a voltage in the range $0.1-10 \mathrm{~V}$ (Keithley, Cleveland, $\mathrm{OH})$. Once the DNA molecules were brought inside the nanochannels, the electric field was switched off and the molecules were allowed to relax to their equilibrium state for $2-5$ min. A fresh chip was used for the measurement of about every 5 molecules and the experiments were done at an ambient temperature of $296 \mathrm{~K}$. Protein and DNA were visualized with a Nikon Eclipse Ti inverted fluorescence microscope equipped with 200-mW/488-nm (YOYO1), $400 \mathrm{~mW} / 556$-nm (Cy3), and $200 \mathrm{~mW} / 640$-nm lasers, a 405/488/561/640-nm filter cube 
(Chroma Technology, Bellows Falls, VT), and 100× oil immersion objective (numerical aperture 1.49). Imaging of DNA and Hfq was facilitated by switching and selecting the relevant laser excitation wavelength for YOYO-1 and Cy3, respectively. Video was recorded with an electron-multiplying charged coupled device camera (Andor iXon X3). The image pixel size of $0.16 \times 0.16 \mu \mathrm{m}^{2}$ was calibrated with the help of a metric ruler. Hfq trajectories, mean square displacements, and diffusion coefficients were obtained with home-developed scripts in MATLAB, R2020b (MathWorks, Natick, MA).

\section{VIDEO CLIPS}

- Clip 1: 1 Hfq on $\lambda$-DNA $\mathrm{DN}_{3}$ trimer.

- Clip 1: 2 Hfq's on $\lambda$-DNA 2 dimer.

[1] J. A. van Kan, A. A. Bettiol, and F. Watt, Proton beam writing of three-dimensional nanostructures in hydrogen silsesquioxane, Nano Lett. 6, 579 (2006).

[2] C. Zhang, F. Zhang, J. A. van Kan, and J. R. C. van der Maarel, Effects of electrostatic screening on the conformation of single DNA molecules confined in a nanochannel, J. Chem. Phys. 128, 225109 (2008).

[3] J. A. van Kan, C. Zhang, P. P. Malar, and J. R. C. van der Maarel, High throughput fabrication of disposable nanofluidic lab-on-chip devices for single molecule studies, Biomicrofluidics 6, 036502 (2012).

[4] R. J. Bauer, A. Zhelkovsky, K. Bilotti, L. E. Crowell, T. C. Evans Jr, L. A. McReynolds, and G. J. Lohman, Comparative analysis of the end-joining activity of several DNA ligases, PloS one 12, e0190062 (2017).

[5] A. Taghbalout, Q. Yang, and V. Arluison, The Escherichia coli RNA processing and degradation machinery is compartmentalized within an organized cellular network, Biochem. J. 458, 11 (2014).

[6] M. Rabhi, O. Espéli, A. Schwartz, B. Cayrol, A. R. Rahmouni, V. Arluison, and M. Boudvillain, The Sm-like RNA chaperone Hfq mediates transcription antitermination at Rho-dependent terminators, EMBO J. 30, 2805 (2011). 